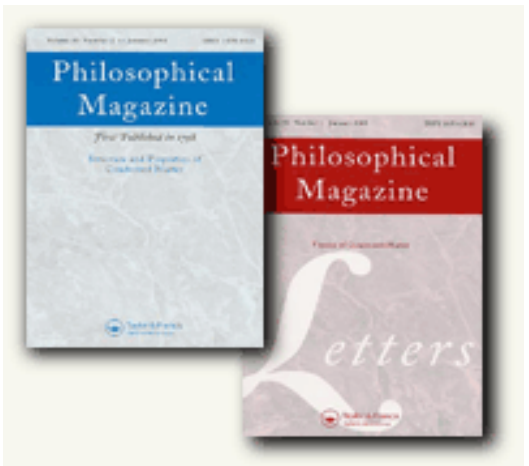

\title{
Investigation of precipitation hardening by dislocation dynamics simulations
}

\begin{tabular}{|r|l|}
\hline Journal: & Philosophical Magazine \& Philosophical Magazine Letters \\
\hline Manuscript ID: & TPHM-06-Apr-0092.R1 \\
\hline Journal Selection: & Philosophical Magazine \\
\hline Date Submitted by the \\
Author: & 02-Jun-2006 \\
\hline Komplete List of Authors: & Monnet, Ghiath; EDF, MMC \\
\hline Keywords: & $\begin{array}{l}\text { dislocation dynamics, mechanics of materials, mesoscopic systems, } \\
\text { plastic deformation, plasticity of metals, precipitation, } \\
\text { strengthening mechanisms, irradiation effects }\end{array}$ \\
\hline Keywords (user supplied): & Orowan, simulation \\
\hline &
\end{tabular}

\section{(5) ScholaroNE \\ Manuscript Central}




\begin{abstract}
Dislocation Dynamics (DD) simulations are used to investigate the precipitation-induced strengthening in a $\mathrm{Zr}-1 \% \mathrm{Nb}$ alloy. A method is proposed to carry out simulations under dynamical conditions in connection with the microstructure of the investigated alloy. First, the sensitivity study to simulation parameters, suspected to alter the simulation results, is presented. It allows setting up simulation conditions ensuring statistical representativeness. The effect of the strain rate is then investigated and analyzed in connection with the random distribution adopted to describe precipitation distribution. The strengthening induced by two different families of $\mathrm{Nb}$ precipitates is estimated from simulations whose simulation box $\underline{\text { is }}$ reduced to the volume of a single grain. It is shown that the so-obtained strengthening values are smaller than predictions by most known published models The determination of the total strengthening shows that usual superposition rules do not apply. A mixture law, fitting well
\end{abstract}




\section{Introduction}

Precipitation hardening is one of the oldest problems of physical metallurgy [1]. Several models have been developed in order to catch the main physical features of the involved dislocation mechanisms. For a review see [2]. In this paper we focus on the case of precipitates of infinite strength causing dislocations to bypass them. The process is widely known as the Orowan mechanism. Analytical and empirical solutions were proposed to describe the critical stress in simple cases, such as uniform and periodic distribution, point obstacles, etc. Even in these cases, models diverge by a factor of 2-3, as will be seen later on. The critical stress can always be put into the general form:

$$
\tau_{c}=\frac{A}{l} \ln D,
$$

where $A$ is the pre-logarithmic term of the dislocation free energy, $l$ the effective spacing of particles pinning the dislocation line, $D$ the dislocation's outer cut-off radius normalized by $b$, the norm of the Burgers vector, taken as an estimation of the dislocation's inner cut-off radius. In the case of a random distribution of impenetrable obstacles, averaging procedures are required for the estimation of $A, l$ and $D$. The pre-logarithmic factor $A$ depends on the dislocation character and does not vary strongly compared to $l$ and $D$ [2]. These two fundamental features depend on the spatial and size distributions [2] and are still difficult to account for.

During the Orowan process, as pointed out by Bacon et al [3], dislocations exhibit two curvatures, one around the precipitate and the second between precipitates. Consequently, $D$ is reduced to the harmonic mean between the precipitate size $d$ and the spacing between precipitates $l . D$ is therefore equal to $l d /[b(l+d)]$. It is worth noting that the approximation leading to the well-known formula of the Orowan stress $\tau_{\mathrm{s}}=\mu b / l$, where $\mu$ is the shear modulus, is obtained by assuming the simple expression of the line tension: $T_{s}=1 / 2 \mu b^{2}$. The subscripted "s" in $\tau_{\mathrm{s}}$ and $T_{s}$ refers to "square" [4] since $\tau_{\mathrm{s}}$ and $T_{s}$ were initially developed for a 
"square" array of particles, i.e. a uniform and periodic arrangement. The exact line tension formula, given by Bacon et al. [3], converges to $T_{s}$ when the value of $D$ approaches 100 . Consequently, when $D$ is different from $100, \tau_{\mathrm{s}}$ does not correspond anymore to the critical stress, even for a square distribution. The model developed by Bacon et al. [3] can hardly be extended to more than one precipitate family. On the other hand, the particle distribution strongly affects the Orowan process [4]. Any deviation from the square array distribution causes the Orowan stress to decrease. From a theoretical point of view, the random distribution is of interest since it offers a simple and well defined [5] alternative to the square distribution. As pointed out by Friedel [6], the average spacing is not equal to the effective spacing of precipitates pinning the dislocation on its line. Friedel reported an original method to estimate the effective spacing which, however, applies only to weak obstacles.

To summarize, theoretical descriptions of the Orowan process are still unsatisfactory and numerical simulations are needed to assess some fundamental issues of the mechanism. The work achieved by Mohles and co-authors, see for example [7,8], on the precipitation hardening in different aging states and using a sophisticated size distribution function $[9,10]$, provides a detailed description of the Orowan mechanism. The method used to generate the microstructure is useful especially for volume fractions larger than $1 \%$ when a complete experimental characterization of the microstructure is available. The method permits precipitates to avoid overlapping and to decrease the distribution randomness. However, these investigations $[7,8]$ were carried out in 2-D using periodic boundary conditions. Their results therefore overestimate the critical stress compared to that obtained in the case of the pure random distribution. In addition, the stress fluctuations during the dislocation glide were not reported. Therefore, it is not possible to confirm that, during these simulations, the system reached a steady state represented by the measured critical stress. 
In this work, we present the first 3-D investigation of the precipitation hardening under dynamical conditions. Two different precipitate families, randomly distributed in a model material, are considered. Investigations are carried out using Dislocation Dynamics (DD) simulations designed for the Hexagonal Close-Packed (HCP) structure. First we present the model material and a study of the sensitivity to simulation parameters and statistical features ensuring the representativeness of DD simulations. Then we report and discuss simulation results in connection with theory.

\section{The Model material: $\mathrm{Zr}-1 \% \mathrm{Nb}$ alloy}

The $\mathrm{Zr}-1 \% \mathrm{Nb}$ alloy is used as cladding material in nuclear power plants [11]. The reference material is characterized by the composition detailed in Table 1.

Table 1

Before irradiation, the alloy contains spherical precipitates of $\beta \mathrm{Nb}$ with mean size $d_{p}=40 \mathrm{~nm}$. The matrix is known to be an over-saturated substitutional solid solution of $\mathrm{Nb}$. During irradiation, the flux of point defects accelerates the $\mathrm{Nb}$ diffusion, inducing precipitation of small needle-like precipitates of $2 \mathrm{~nm}$ thickness and almost $6 \mathrm{~nm}$ length. For a review see [12]. Needles were found to lay roughly in the basal plane. Experimental investigation [11] allowed the determination of the volume densities: $Q_{p}=10^{20} \mathrm{~m}^{-3}$ and $Q_{n}=10^{22} \mathrm{~m}^{-3}$, where indices " $n$ " and " $p$ " refer to needles and particles, respectively. It is worth noting that the large spherical precipitates are not affected by irradiation, neither in size nor in density. Irradiation generates also large densities of prismatic dislocation loops, which are suspected to induce large strengthening. They are not considered in this work and only the precipitationstrengthening component is investigated. 
In the following, for the sake of brevity, the spherical $\mathrm{Nb}$ precipitates are named particles and the thin $\mathrm{Nb}$ needles appearing under irradiation are called simply needles. The term "precipitates" is used as a generic term in the discussion to designate both families.

We assume that the intrinsic mechanical behavior of the matrix is similar to that of single crystals $[13,14]$ with the same oxygen content. Other alloying elements in solid solution, see Table 1, do not strengthen the matrix, especially at high temperature, because of their small content. Tensile tests performed on single crystals of $\mathrm{Zr}-1200 \mathrm{ppm}$ wt of oxygen [15] allowed to determine the Critical Resolved Shear Stress (CRSS) as a function of temperature. An athermal plateau [16] is observed for T $>580 \mathrm{~K}$, corresponding to a CRSS of almost 20 MPa. We may assume then that the alloy friction stress, $\tau_{F}$, characteristic to this alloy is 20 MPa. All simulations in this work are performed at $600 \mathrm{~K}$ where the behavior is athermal, i.e. the lattice friction is completely overcome by thermal activation.

The main idea in this work is to quantify first the precipitation strengthening due to the particles in order to estimate, $\tau_{p}$, the critical stress before irradiation and, then, to evaluate, $\tau_{r}$, the critical stress after irradiation. Since the state of the initial spherical precipitates is not altered by irradiation, $\tau_{r}$, therefore, accounts for the presence of both precipitate families: particles and needles.

Although the precipitation of needles occurs in the presence of particles, it is interesting from a theoretical point of view to evaluate the precipitation strengthening resulting from the presence of needles only. The associated critical stress is denoted $\tau_{\underline{n}}$. All precipitates are assumed to have a $\mathrm{BCC}$ structure [11] while the matrix is of $\mathrm{HCP}$ structure. Dislocations are thus assumed to bypass both particles and needles in accordance with the Orowan process. This process is not thermally activated because of the large associated activation volumes ( $>100 b^{3}$ ). The strengthening is thus temperature independent.
Deleted: $[\mathrm{O}]$ single crystals

Deleted: particle family in

Deleted: be of 


\section{Method and simulation technique}

Readers interested by a description of the DD simulations and the numerical method are invited to consult [17]. For more details about the method used to account for the hexagonal symmetry and slip system activity of the HCP structure, readers are referred to [18].

We present here only numerical choices made to account for the special physical features related to the Orowan mechanism. When cross-slip and dislocation climb are negligible, the quasi-static component of the precipitation hardening is a pure two-dimensional phenomena. This is why most treatments reported in the literature on the Orowan process were 2D approaches. In this work, we introduce dynamics by applying a fixed strain rate through 3D simulations.

\subsection{Representation of precipitates in the simulation space}

Precipitates are represented by spherical volumes in the simulation space. To every spherical volume, a friction stress, $\tau_{\text {shear }}$, is attributed in order to account for the resistance of the precipitate to dislocation penetration. $\tau_{\text {shear }}$ always opposes the dislocation movement in the same manner as the alloy friction introduced above. This representation is appropriate for DD simulations because $\tau_{\text {shear }}$ can readily be compared to the effective stress computed on the segment attempting to enter the precipitate. Therefore, it allows avoiding the concepts of pinning force and critical angle, which imply the use of an isotropic line tension model Of course, if any comparison with the line tension model is needed, the link with the classical pinning force, $F_{c}$, can be readily given: $F_{c}=b d \tau_{\text {shear }}$, where $d$ is the precipitate size. 
Only a precipitate cutting the slip plane of a dislocation constitutes an obstacle. Thus the number of precipitates introduced in the 3D simulation box can be reduced to the number of precipitates cutting planes where dislocations where introduced. This leads us to consider the surface density $C$ in our DD simulations. The connection with the volume density, $Q$, can be given $C=d Q$. In practice, the parameter used to deduce the number of precipitates is the average spacing between precipitates, $l$, which is also the fundamental parameter in theoretical models. $l$ can be deduced directly from the volume density. It is roughly equal to: $l=(d Q)^{-0.5}=C^{0.5}$.

\subsection{Dislocation mobility law}

In the athermal plateau, i.e. $\mathrm{T}>580 \mathrm{~K}$, the lattice friction is completely overcome by thermal activation $\left.\_18\right]$. We assume then that a unique mobility law can describe the mobility of dislocations of all types of characters:

$$
v=\left\{\begin{array}{ll}
0 & \left|\tau_{\text {eff }}\right|\left\langle\tau_{F}\right. \\
B\left(\tau_{\text {eff }}-\operatorname{sign}\left(\tau_{\text {eff }}\right) \tau_{F}\right) & \left|\tau_{\text {eff }}\right| \geq \tau_{F}
\end{array},\right.
$$

where $v$ is the dislocation velocity, $\tau_{e f f}$ the effective stress computed in DD simulations and $B$ a viscous drag coefficient. When any portion of a segment is located inside the interaction volume of a precipitate $\tau_{F}$ is replaced by $\tau_{\text {shear }}$ in the mobility law. By definition, in the case of the Orowan process, the penetration of the dislocation is impossible, fixing the value of $\tau_{\text {shear }}$ to infinity. This causes the dislocation to bypass the interaction area leaving a dislocation loop around it.

\section{Sensitivity to simulation parameters}

Two simulation parameters are suspected to affect the critical stress computed in DD simulations: (i) the initial dislocation length, i.e. the size of the Frank-Read source, which 
determines roughly the total number of pinning events on dislocations, and (ii) the initial dislocation character, known to affect strongly the bypassing process [3].

\subsection{Statistics and initial dislocation length}

A rough estimate of the number, $N$, of precipitates pinning simultaneously the dislocation can be obtained by $N=L / l$, where $L$ is the total length of mobile dislocations. From a statistical point of view, $N$ is the most important parameter of the process. First, simulations are dedicated to estimate a realistic value of $N$, ensuring a good representativeness of DD simulations. Two separate simulations differing only in the length of the Frank-Read source, 4 and $40 \mu \mathrm{m}$, are performed. The simulation box size is $(60 \mu \mathrm{m})^{3}$ and contains the same number of precipitates. The precipitate distribution used for this test is that of particles: $d_{p}=40 \mathrm{~nm}$ and $l_{p}=0.45 \mu \mathrm{m}$. An important aspect has to be considered here. If one wants to conserve the same dynamic conditions of the Orowan mechanisms in both simulations, one has to impose the same average velocity, $v$, of the dislocation. Since $v$ is related to the strain rate, $\dot{\gamma}$, by the Orowan relation, $\dot{\gamma}=\rho b v$, where $\rho$ is the mobile dislocation density, the strain rate should be adjusted dynamically and proportionally to the dislocation density $\rho$. The initial strain rates were fixed to 0.01 and $0.001 \mathrm{~s}^{-1}$ in simulations with $L=40$ and $4 \mu \mathrm{m}$ respectively. The corresponding stress-strain curves are shown in Figure 1.

Figure 1

It can be shown that a simulation with $L=40 \mu \mathrm{m}$ leads to an almost monotonous loading. The shear stress stabilizes when it reaches the value of $32 \mathrm{MPa}$, while the stress fluctuates strongly in the simulation with $L=4 \mu \mathrm{m}$. A large yield point is observed at the beginning of the plastic deformation followed by a large decrease in stress. Furthermore, the fluctuations tend to slow

Deleted: strong Deleted: ing Deleted: But 
down and their magnitude decreases with increasing strain. Both simulations lead, however, to the same saturation stress value of $32 \mathrm{MPa}$. This convergence can be readily explained. On the one hand, plastic deformation is proportional to the swept area by the dislocation and, on the other hand, the dislocation length is roughly proportional to the square root of the swept area. Consequently, in order to achieve the same deformation, dislocation density in both simulations must converge to the same value. We can roughly estimate the number of obstacles interacting simultaneously with dislocations, $N$. When the shear strain reaches $810^{-7}$, the surface scanned by the dislocation is the same in both simulations and the dislocation total length becomes roughly the same. Therefore, for large shear strains the dislocation densities and the flow stresses are almost the same. In this steady state, the lower limit of $N$, can be evaluated. The swept area by the dislocation at a given $\gamma$, is equal to $V \gamma / b$, where $V$ denotes the simulation box volume. For an initially small FR source and a large swept area, the generated dislocation loop edging the swept area can be assimilated to a square of an equivalent area. If the total length of this loop is divided by the average spacing of particle, we get a rough estimation of the lower limit of $\mathrm{N}$ :

$$
N \geq \frac{4}{l} \sqrt{\frac{V \gamma}{b}}
$$

Application of equation 3 results in a value of $N$ close to 200. Consequently, we can establish a criterion ensuring the statistical representativeness of our simulation: the total length of dislocations interacting with precipitates should be at least equal to 200 times the average spacing of precipitates.

\subsection{Effect of the initial dislocation character}

In $\mathrm{Zr}$, where the Poisson's ratio is $v=0.3\lceil 19]$, the line tension of screw dislocation is almost 4 times larger than that of edge dislocations [20]. One may suspect then the initial character of 
the FR source to play an important role. Therefore, 3 simulations were carried out with screw, edge and $58.5^{\circ}$ mixed dislocation characters and the corresponding stress-strain curves are shown in Figure 2.

Figure 2

The same particle distribution is used jn this study. The initial length of FR sources is $6 \mu \mathrm{m}$. The strain rate is fixed to $10^{-4} \mathrm{~s}^{-1}$ in all simulations, which explains the high frequency of stress fluctuations. Although the criterion established in the last section is not fulfilled, we note that the stress in Figure 2 fluctuates in all simulations about the same average value with almost the same fluctuation amplitude. This indicates the presence of local events causing stress fluctuations, which are independent of the initial dislocation character. Observing dislocation behavior in DD simulations allows us to correlate these events to bursts corresponding to the bypassing of several precipitates. The dislocation thus selects paths in the slip plane where the bypassing process is possible under the present mechanical loading. The character effect is therefore closely associated to the local precipitate distribution. When the dislocation is long enough, the presence of these paths dilutes the effect of the initial character of the Frank-Read source. To illustrate this, we show in Figure 3 the dislocation configuration corresponding to the $40 \mu$ m-dislocation simulation.

Figure 3

The directions of favored paths, pointed out by arrows, are independent from the initial dislocation character. In most cases dislocation segments participating in the bypassing bursts are of mixed character even when the initial character is screw, see Figure 3 . This is why the 


\section{DD simulation results}

The previous section allows us to set up DD simulation conditions for the determination of precipitation hardening before and after irradiation. The study of sensitivity to simulation parameters shows that the initial length of the Frank-Read source must be at least 200 times larger than the average spacing of precipitates and that under this condition the initial character of the FR sources does not affect the critical stress.

\subsection{Effect of strain rate on the Orowan process}

The general treatment in quasi-static simulations $[4,7]$ consists in increasing the stress as long as the dislocation is not able to bypass most of precipitates in the simulation box. In dynamical simulations, the stress increment is dynamically adjusted as a function of the fixed strain rate. When the strain rate computed in DD simulation is less than the imposed one, the stress is increased. Otherwise, the stress is decreased. Therefore, in dynamical conditions, the stress fluctuates depending on the temporary state of the system. Hard configurations cause the stress to increase until reaching local maxima. In order to investigate this property, two simulations were carried out in identical condition except for the strain rates, see in Figure 4.

The initial length of the Frank-Read source is $40 \mu \mathrm{m}$ with a random distribution of particles. 
The two different strain rates are 1 and $0.1 \mathrm{~s}^{-1}$. It will be seen in the following sections that only the relative value of the strain rate is significant here.

Figure $\underline{4}$

Two remarks can be made from Figure 4: the flow stress increases and the amplitude of stress fluctuation decreases with increasing strain rate. This tendency can be explained as follows. For small strain rates, when a favorable path is reached (see Figure 3), the dislocation becomes locally unstable; the measured strain rate exceeds the imposed one, leading to a decrease of the stress. This is the origin of stress fluctuations on the stress-strain curve in dynamical simulations. An increase of strain rate causes the stress to increase in order to allow the dislocation to take a larger number of favored paths. But an increase of the number of active paths reduces stress fluctuations and smoothes the stress-strain curve. Consequently, the critical stress in the quasi-static response represents the lowest limit for the critical stress of the Orowan mechanism. Precipitation strengthening predicted in DD simulations should in principle be larger than that yielded by quasi-static simulations $[3,4]$. However, this is not the case as discussed in the following.

\subsection{Determination of precipitation strengthening in $\mathrm{Zr}-\mathrm{Nb}$ alloys}

As discussed in the last section, the measured Orowan stress in a large crystal in dynamical conditions depends on the applied strain rate. This dependency results from the distribution randomness. Restriction of this randomness decreases the sensitivity to the strain rate. In real materials, the random distribution is naturally restricted by several factors, such as grain boundaries and cooling rate, which reduces the diffusion distance of solute atoms. In a $\mathrm{Zr}$ $1 \% \mathrm{Nb}$ alloy, the grain size is close to $4 \mu \mathrm{m}$. It is thus realistic to consider that the random 
1

2

3

4

5

6

7

8

9

10

11

12

13

14

15

16

17

18

19

20

21

22

23

24

25

26

27

28

29

30

31

32

33

34

35

36

37

38

39

40

41

42

43

44

45

46

47

48

49

50

51

52

53

54

55

56

57

58

59

60 distribution is restricted to a volume of $(4 \mu \mathrm{m})^{3}$. The following simulations are dedicated to simulate the precipitation hardening in an individual grain of the alloy with and without needles, Considering that dislocation density on the active slip system is close to $2.510^{11} \mathrm{~m}^{-2}$, the initial length of dislocations is thus close to $16 \mu \mathrm{m}$, distributed on 10 slip planes. The total length of Frank-Read sources in DD simulation was set to $16 \mu \mathrm{m}$.

The strain rate is fixed to $0.3 \mathrm{~s}^{-1}$. We were not able to perform massive DD simulations at lower strain rates, because of prohibitive simulation times. Precipitates are distributed only on populated slip planes, which fixes the number of particles in the simulation box to 1085 . In the simulation with needles the number of needles is thus 11283. Figure 5 shows the corresponding shear stress-shear strain curves for the two simulations, performed with the same conditions.

Figure 5

The thin line represents the simulated loading curve of the alloy without needles, i.e. when the simulation box contains only the 1085 spherical particles. The alloy friction stress is fixed to $20 \mathrm{MPa}$ [15], see Section 2. Therefore, particle strengthening corresponds to almost $12 \mathrm{MPa}$. In order to simulate the precipitation hardening with needles, (thick line), the population of needles is added to the particle family in the simulation box. The total precipitation strengthening, i.e. after subtracting the solid solution threshold corresponds to 20_MPa. In Figure 5, we can see also the loading curve corresponding to a fictive material, containing only the needle population. This last simulation is performed for comparison with theory and is of theoretical interest as discussed in the following section.
Deleted: before

Deleted: after irradiation

Deleted: of the irradiated grain

Deleted: non-irradiated

Deleted: the

Deleted: in the irradiated material 


\section{Discussion}

As a basis of our discussion, we compare in Table 2 our results given in Figure 5 with predictions of some analytical models available in the literature: Orowan [22], Kocks [23], Foreman et al [4] and Bacon et al. [3]. $\tau_{\mathrm{c}} / \tau_{\mathrm{s}}$ denotes the ratio between the predicted stress and the square array stress $\tau_{\mathrm{s}}=\mu b / l . \Phi$ is an angle parameter designed in the model developed by Bacon, Kocks and Scattergood [3], called the BKS model in the following. The absolute value of $\Phi$ is important since it accounts for the precipitate size effect and allows the authors to use the Friedel statistics [6]. This approach applies only for weak obstacles, i.e. when $\Phi$ is large enough, say more than $80^{\circ}$. We note here that since the predictions of the first three models scale as $\tau_{\mathrm{s}}$, the relevant information is therefore the ratio $\tau_{\mathrm{c}} / \tau_{\mathrm{s}}$ given in Table 2 . We give also the predictions of precipitation hardening in the case of particles, needles and, when possible, of the presence of both precipitate families. More details can be found in the above mentioned references.

Table 2

As shown in Table 2, $\tau_{\mathrm{s}}$, given by the Orowan model [22] represents an upper bound of all estimates. All other models yield smaller values by accounting for either the randomness of the distribution or the decrease of the line tension due to the dipolar interaction. Nevertheless, compared to DD simulation, all models overestimate the critical stress. These predictions, all of quasi-static type, are still larger than those given in DD simulations under a relatively large applied strain rate $\left(0.5 \mathrm{~s}^{-1}\right)$. Furthermore, this difference between simulations and theory should in principle increase if the strain rate in our simulation is decreased to $10^{-4} \mathrm{~s}^{-1}$, which is the usual value used in experiments. Hence, these models substantially overestimate the critical stress of the Orowan process. 
On the other hand, the difference between DD and theory is larger in the case of needles. The explanation resides in the specific value of $D$, which equals 114 and 12 , in the case of particles and needles, respectively. For needles, this value is too far below the reference value 100 , necessary to derive $\tau_{\mathrm{s}}$, see Introduction. This is why, the predictions made in $[22,23,4]$ for particles are relatively better than those for the needle family. But, even when the value of $D$ is very close to 100 , the deviation will persist because of the randomness of the distribution, which is only partially accounted for in theory.

In Table 2, we note that the BKS model provides a good description for both families. Bacon, Kocks and Scattergood [3] were the first to point out that the precipitate size modifies the logarithmic term of the line tension because of the variation of the outer cut-off radius of the elastic field of bowing dislocations. In this model, one can find also a subtle approach using the Friedel statistics [6]. However, the BKS model is developed for a constant size of precipitates and does not apply directly to a structure involving two precipitate families, In this last case, one may try a simple extension of the BKS model. The harmonic mean $D$ can be taken equal to the average of harmonic means for particles and needles, weighted by their respective density. On the other hand, the average spacing $l$ can be calculated from the sum of the densities of particles and needles. This leads to $D=21, l=0.13 \mu \mathrm{m}, \Phi=117^{\circ}$ and finally $\tau_{\mathrm{c}}=27 \mathrm{MPa}$ for the BKS model. This value does not fit the present DD results. This simple generalization is not appropriate, at least when the characteristic $D$ values are very different. Now let us look for a simple mixture law, which fits well DD results. Before doing this, we should account for the difference between $\tau_{\mathrm{s}}$ and $\tau_{\mathrm{c}}$ calculated by DD simulations for each and every precipitate family taken alone. In all cases, one can write a general formula for precipitation strengthening $\tau_{i}$ related to the precipitate family " $i$ ":

$$
\tau_{i}=\beta_{i} \frac{\mu b}{l_{i}}
$$

Deleted: the irradiated microstructure, i.e. when both families exist 
where $\beta_{i}$ is a variable usually associated with the strength of the precipitate family " $i$ ", since it accounts for the deviation from the square array stress $\tau_{\mathrm{s}, i}=\mu b / l_{i}$. As pointed out in the BKS model, $\beta_{i}$ decreases with decreasing $D_{i}$, the harmonic mean associated with the family " $\mathrm{i}$ ". Since $\underline{\tau}_{\underline{s}, \underline{i}}$ is always substantially smaller than the critical stresses, $\beta_{i}$ is always less than unity and the Orowan process can be pictured as if the precipitates were of finite strength and could be sheared by the dislocation. Using this concept, $\beta_{l}$ can be simply estimated from the ratio between the critical stress and $\underline{\tau_{S, i}}$. Here we can note that $\tau_{i}$ can be determined from DD simulations or by application of the BKS model. The question to answer is how different strengthening $\left(\tau_{i}\right)$ should be superimposed. In other terms how the total strengthening $\tau_{c}$ is related to $\left(\tau_{i}, l_{i}\right)$ ?

In the literature, several rules were proposed in different cases [24,25] that are all based on expressions of the from: $\tau_{c}^{k}=\sum \tau_{i}^{k}$ and differ by the value of the power $k$. Two laws were proposed for two simple cases: (i) all precipitates are characterized by the same strength parameter $\beta_{i}$ [26]. In this case, precipitate densities are added and the quadratic average is found: $k=2$. (ii) When the density of all precipitate families is the same, i. e. same $l_{i}$, Labusch [27] proposed another superposition rule in which the average is taken with $k=3 / 2$.

Neither of these approaches can be used in the present case, since densities and strengths are significantly different.

We propose another mixture law of the form:

$$
\tau_{c}=\beta \frac{\mu b}{l}
$$

where $\beta$ and $l$ are variables to be determined appropriately as functions of $\beta_{\mathrm{i}}$ and $l_{\mathrm{i}}$. We propose to deduce $\beta$ from the average of the $\beta_{\mathrm{i}}$ values weighted by their respective surface densities: 
Equation (7) gives the critical stress as a function of the average spacing or surface densities of different precipitate families and their respective strengths. In the case of two precipitate families, equation (7) reduces to:

$$
\tau_{c}=\frac{\tau_{p} l_{n}+\tau_{n} l_{p}}{\sqrt{l_{n}^{2}+l_{p}^{2}}},
$$

where the subscripts "p" and " $\mathrm{n}$ " hold for particles and needles. With $l_{\mathrm{p}}=0.45 \mu \mathrm{m}, l_{\mathrm{n}}=0.14$ $\mu \mathrm{m}, \tau_{\mathrm{p}}=12 \mathrm{MPa}$ and $\tau_{\mathrm{n}}=18 \mathrm{MPa}$, equation (8) yields $\tau_{\mathrm{c}}=20.5 \mathrm{MPa}$, which is almost the same value as the one computed from DD simulations, see Table 2. Equation (8) represents a mixture formula that is obtained altering artificially the concentrations of the different precipitate families. The mixture law in equation 7 is thus conservative. Equation (8) is equivalent to the law of mixtures proposed by Brown and Ham's [28]. It reduces to the quadratic average [26] when all precipitates families are characterized by the same value of $\beta$. In this case the common strength parameter $\beta$ vanishes and equation (8) reduces to $\tau_{c}=$ $\sqrt{\tau_{n}^{2}+\tau_{p}^{2}}$. On the other hand, when the precipitate families have the same concentration $C_{i}$, equation (8) simplifies to $\tau_{c}=1 / \sqrt{2}\left(\tau_{n}+\tau_{p}\right)$, which is close to the linear superposition rule 
[29], but, however, in disagreement with Labush's superposition rule [27]. Further DD simulations should be carried out in order to investigate these asymptotic configurations.

\section{Conclusions}

DD simulations are a powerful tool to investigate the Orowan stress. Without any adjustable parameter, this method provides precise estimates for the critical stress under satisfactory statistical conditions. Applying a fixed strain rate allowed us to monitor the stress stability and to correlate the stress fluctuations to the statistical features of the dislocation-precipitates interactions. These simulations led to the following main results.

- When the total dislocation length in the active slip systems is much larger than the average spacing of precipitates, the initial dislocation character does not affect the Orowan stress.

- In the case of a low volume fraction of precipitates, the bypassing procedure is controlled by the presence of favorable paths related to local density fluctuations.

- In dynamical loading conditions, the magnitude of the stress fluctuation depends on the value of the imposed strain rate coupled to the size of the simulated volume. An increase in the strain rate smoothes the stress curve and increases the critical Orowan stress. compared to existing models. DD simulations show that the radiation-induced hardening related to the precipitation of $\mathrm{Nb}$ needles is close to $25 \%$. This result clearly indicates that radiation-induced precipitation is not responsible for the large hardening of irradiated $\mathrm{Zr}$ $1 \% \mathrm{Nb}$ alloys.

- DD simulation results show that all models, except the BKS model [3], overestimate the Orowan stress even with respect to DD simulation carried out at large strain rates.

Deleted: When a random distribution is generated in a large volume, strong fluctuations of precipitate density can be observed locally. The number and the magnitude of these fluctuations increases with the simulated volume. 
- A general superposition rule is proposed to account for the strengthening induced by different precipitate families characterized by different densities and strengths.

\section{Acknowledgment}

This work was partly supported by the European project SIRENA (SIR1-CT-2001-20137). 


\section{Reference}

[1] Mott N.F. and Nabarro F.R.N., Proc. Phys. Soc., 5286 (1940)

[2] Nembach E., in "Particle Strengthening of Metals and Alloys", J. Wiley \& SONS, New York, 1997.

[3] Bacon D.J., Kocks U.F. et Scattergood R.O., Phil. Mag., 281241 (1973).

[4] Foreman A.J.E. et Makin M.J., Phil. Mag., 13911 (1966).

[5] Asim Tewari, A.M. Gokhale, Materials Science and Engineering A 385332 (2004).

[6] Friedel J., "Dislocations”, Pergamon ; 31 (1964).

[7] Mohles V., Rönnpagel D. et Nembach E., Comp. Mat. Sci., 16144 (1999).

[8] Mohles V. et Nembach E., Acta Mater., 492405 (2001).

[9] Wagner C., Z. Electrochem., 65581 (1961).

Formatted: English (U.K.)

[10] Lifshits I.M. et Slyozov V.V., J. Phys. Chem. Sol., 1935 (1961).

[11] Doriot S., Jilbon D., Bechade J.L., Mathon M., Legras L. and Mardon J.P., publishing in progress in J. Test. and Eval..

[12] Griffiths M., J. Nuc. Mat., 159190 (1988).

Formatted: French (France)

[13] Rapperport EJ. and Hartley CS., Trans Metall Soc AIME, 218869 (1960).

Formatted: French (France)

[14] Akhtar A., Met Trans A, 61217 (1975).

[15] Mills D. and Craig GB. Trans Metal Soc AIME, 2421881 (1968).

[16] Tang M, Kubin LP. and Canova GR., Acta Mater, 463221 (1998).

[17] Devincre B., Kubin L., Lemarchand C. and Madec R., Mater. Sci. Eng. A, 309-310, 211 (2001).

[18] Monnet G., Devincre B. and Kubin L.P., Acta Mater., 524317 (2004).

[19] Simmons G., Wang H., Single Crystal Elastic Constants and Calculated Aggregate Properties: A Handbook, MIT, Cambridge, 1971.

[20] De Wit G. and Koehler JS., Phys Rev, 1161113 (1959).

[21] Hirsch PB. and Humphreys FJ, Physics of Strength and Plasticity, ed. A. Argon, MIT Press, Cambridge, Massachusetts (1969), 189. 
[22] Orowan E., Symposium on Internal Stresses in Metals and Alloays, London : The Institute of Metals, 451 (1948).

[23] Kocks U.F., Can. Jour. Phys., 45737 (1967).

[24] Kocks U.F., A.S. Argon and M.F. Ashby, Prog. Mat. Sci., 191 (1975).

[25] U. Lagerpusch, V. Mohles and E. Nembach, Mat. Sci. Eng. A319-321, 176 (2001).

[26] Koppenaal T.J. and Kuhlmann-Wilsdorf D., Appl. Phys. Letters, 459 (1964)

Formatted: French (France)

[27] Labusch R., Phys. Stat. Sol., 41659 (1970).

Formatted: French (France)

[28] Brown LM. And Ham RK., in Strengthening Methods in Crystals, ed. A. Kelly and R.B. Nicholson, Applied Science Publishers, London 1971, p. 9.

[29] Ebeling R. and M.F. Ashby, Phil. Mag., 13805 (1966). 


\section{Tables}

\begin{tabular}{|c|c|c|c|c|c|c|}
\hline $\mathrm{O}$ & $\mathrm{Nb}$ & $\mathrm{S}$ & $\mathrm{Fe}$ & $\mathrm{Cr}$ & $\mathrm{Sn}$ & $\mathrm{V}$ \\
\hline 1150 & 9700 & 33 & 237 & 32 & $<30$ & $<10$ \\
\hline
\end{tabular}

Table 1: chemical composition in wt ppm of the $\mathrm{Zr}-1 \% \mathrm{Nb}$ alloy.

\begin{tabular}{|c|c|c|c|c|}
\hline Model & $\tau_{\mathrm{c}} / \tau_{\mathrm{s}}$ & $\tau_{\mathrm{c}}$ (particles) & $\tau_{\mathrm{c}}$ (Needles) & $\tau_{\mathrm{c}}$ (all) \\
\hline Orowan [20] & 1 & 17 & 56 & 59 \\
\hline Kocks [21] & 0.84 & 14 & 47 & 50 \\
\hline Foreman et al. [4] & 0.81 & 14 & 45 & 47 \\
\hline Bacon et al. [3] & Not proportional & $13\left(\Phi=83^{\circ}\right)$ & $20\left(\Phi=117^{\circ}\right)$ & - \\
\hline DD simulations & Not available & 12 & 18 & 20 \\
\hline
\end{tabular}

Table 2: Prediction of theoretical models of precipitation strengthening compared to DD results. All critical stress are given in MPa. The shear modulus of this alloy used in DD simulation is equal to $24.5 \mathrm{GPa}$. 


\section{Figure Captions}

Figure 1: Effect of the initial length of the Frank-Read source on the stress-strain curves under a dynamically adjusted strain rate.

Figure 2: Effect of the dislocation character on the critical stress of the Orowan mechanism with a large number of precipitates.

Figure 3: typical shape of dislocation during the Orowan process. The initial Frank-Read source is of screw character. Arrows indicate local paths taken by the dislocation along directions different from the initial direction of the dislocation line.

Figure 4: Influence of the strain rate on the stress-strain curve in the case of the particles.

Figure 5: Shear stress - shear strain curves at a strain rate of $0.5 \mathrm{~s}^{-1}$ for three precipitation states: particles (thin line), needles (intermediate line) and both families (thick line). 
Figures

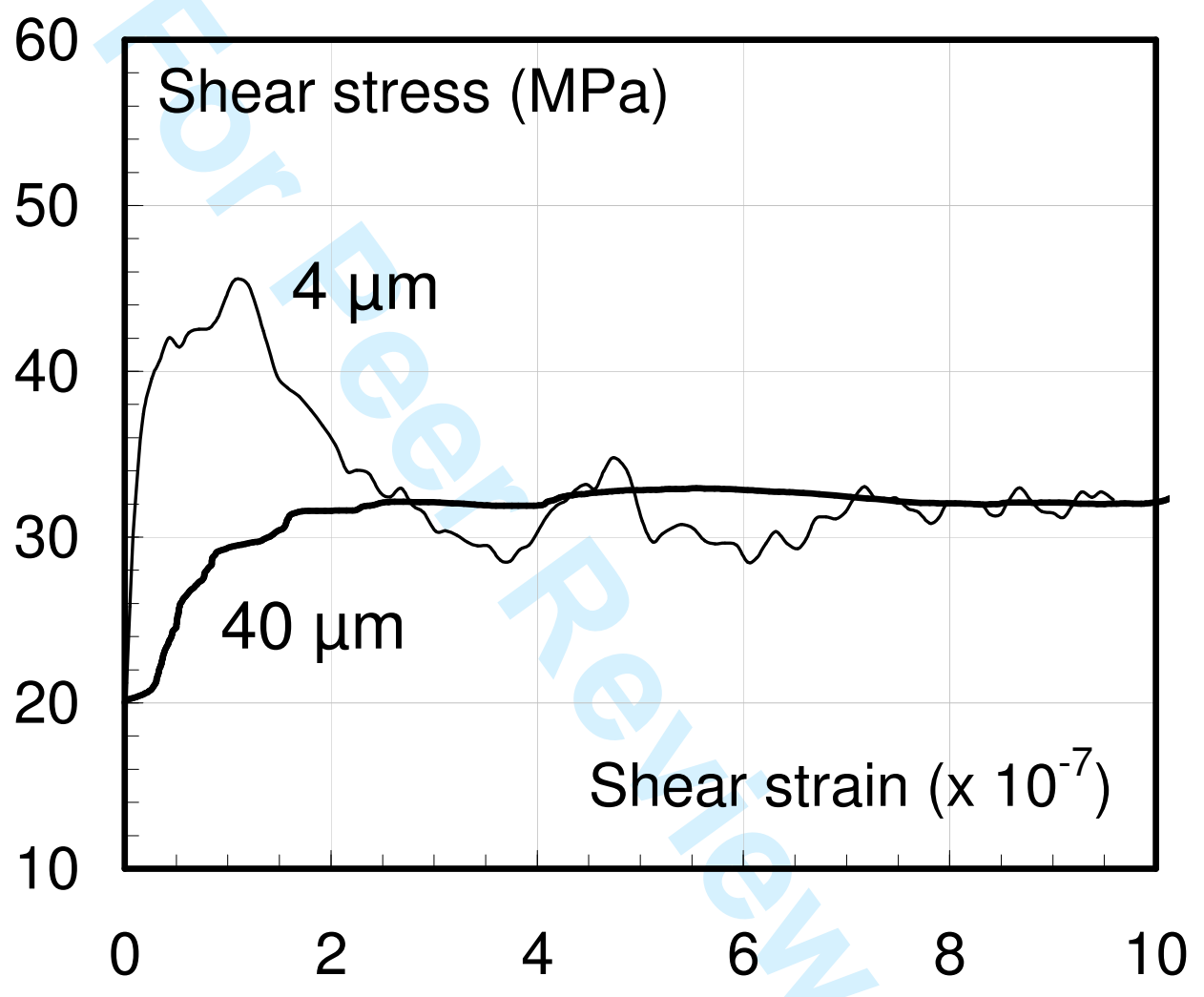

Figure 1 


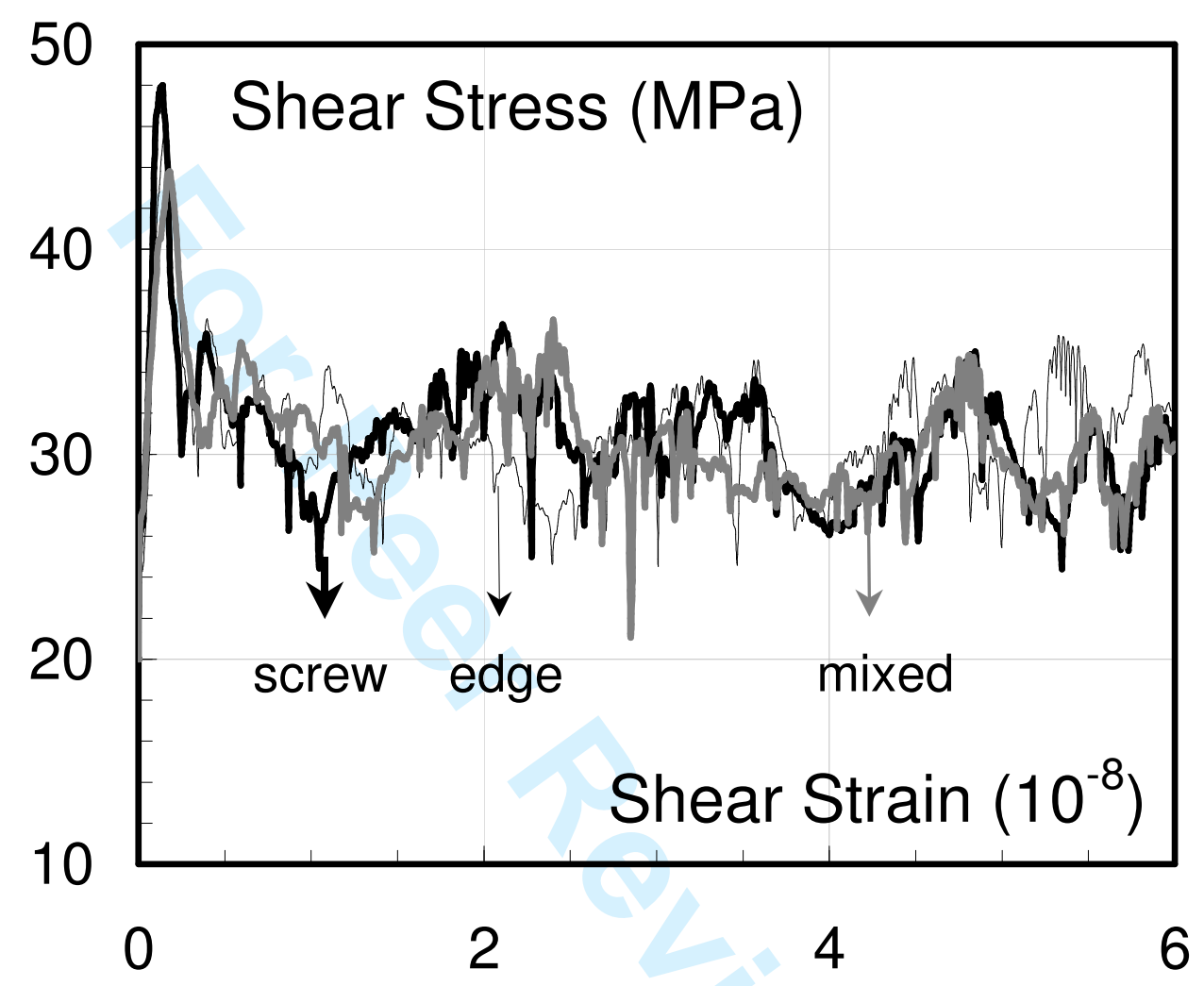

Figure 2 




Figure 3 




Figure 4 


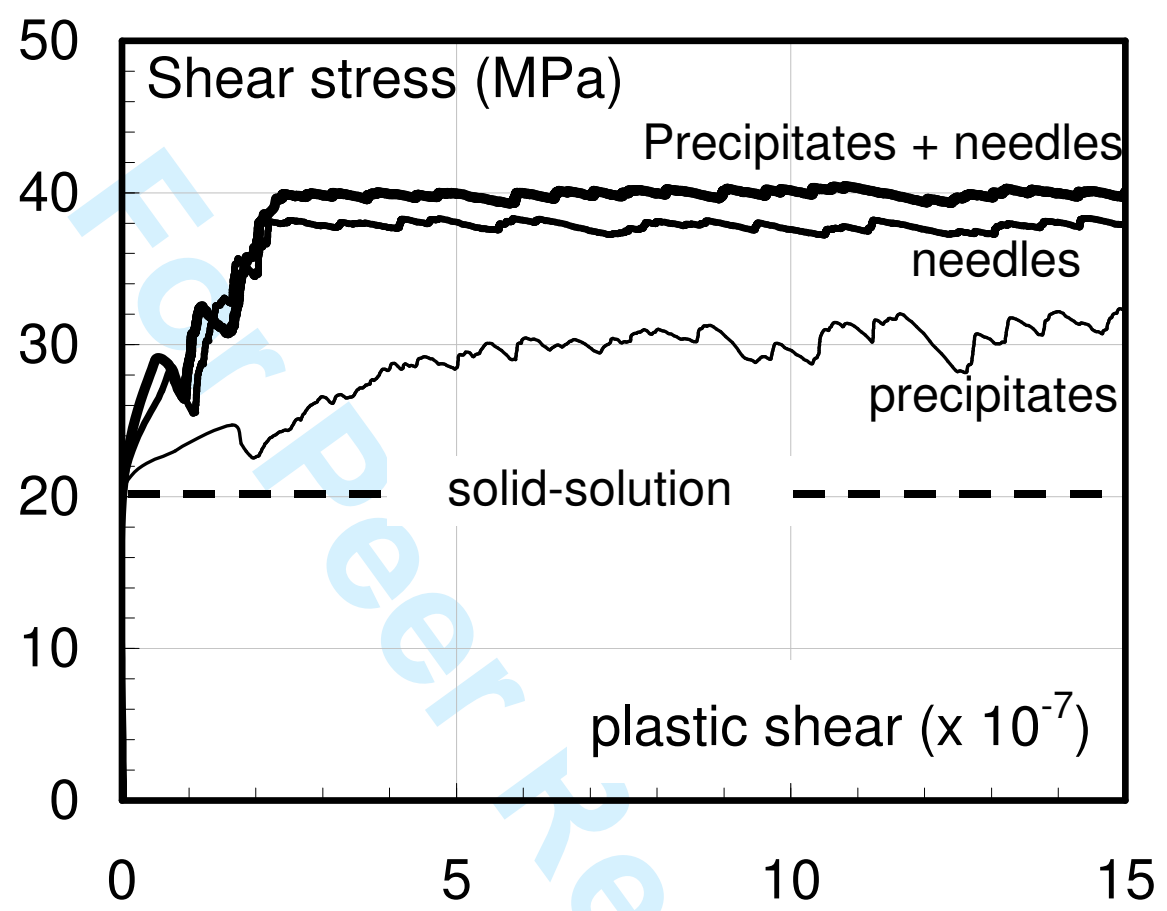

Figure 5 